\title{
Evaluation of the impact of a clinical pharmacist on a specialty neurology clinic's adherence to recommended laboratory test monitoring
}

Sarah Darby, PharmD, BCPS, and Pamela J Mazyck, PharmD, MSCR, BCPS, BCACP, CDE

\section{What is already known about this subject}

- Patients requiring specialty medications often have complex disease states and require high levels of care.

- Use of specialty medications requires frequent monitoring and evaluation and may place patients at high risk for adverse events.

- Pharmacists may work collaboratively with other health care providers in the specialty setting to optimize safe and effective medication therapy and improve patient outcomes.

\author{
What this study adds \\ - Incorporation of a pharmacist in a \\ specialty neurology clinic may lead to \\ improved laboratory test monitoring of \\ high-risk medications. \\ - A collaborative drug therapy \\ management agreement that allows \\ for pharmacist-conducted laboratory \\ test monitoring may improve the care \\ provided to patients with neurological \\ disorders.
}

\author{
Author affiliations \\ Sarah Darby, PharmD, BCPS, University \\ Health Network, University of Tennessee \\ Medical Center, Knoxville, and Pamela \\ J Mazyck, PharmD, MSCR, BCPS, BCACP, \\ $\mathrm{CDE}$, Medical University of South Carolina, \\ Charleston.
}

AUTHOR CORRESPONDENCE: Sarah Darby, 865.471.8184; sdarby@utmck.edu

J Manag Care Spec Pharm. 2021;27(12):1664-70

Copyright $@ 2021$, Academy of Managed Care Pharmacy. All rights reserved.

adherence rates were compared using chisquare or Fisher's exact tests.

METHODS: This study was a retrospective chart review at a single academic medical center for the period between July 1, 2014, and April 30, 2020, comparing laboratory test monitoring adherence before (prepharmacist) and after (post-pharmacist) incorporation of a pharmacist into a neurology clinic. Patients were included if they lived in the Tri-County Area of Charleston, South Carolina, and received a prescription for dalfampridine, dimethyl fumarate, fingolimod, teriflunomide, or cannabidiol that was prescribed by a neurology clinic provider at the Medical University of South Carolina. Chart review was conducted to assess clinic adherence with manufacturer-recommended laboratory test monitoring. Laboratory test monitoring was considered adherent if obtained within 6 months before or on the date of prescription order. Descriptive statistics were calculated for all variables, and
RESULTS: For dalfampridine, dimethyl fumarate, fingolimod, and teriflunomide, there were 123 patients and 78 patients in the preand post-pharmacist groups, respectively. There were 51 patients in the cannabidiol group. Clinic adherence to laboratory test monitoring improved in the post-pharmacist group for every monitoring point, with statistically significant improvement in "hepatic function tests every 6-9 months" ( $P=0.005)$, "CBC every 6-9 months" $(P=0.01)$, and "VZV IgG titer at baseline" $(P=0.005)$ for patients taking fingolimod.

CONCLUSIONS: Our study demonstrated improved adherence to manufacturer-recommended laboratory test monitoring after a specialty clinical pharmacist was incorporated into a multidisciplinary neurology clinic. 
Pharmacists who practice in specialty ambulatory clinics care for patients with complex disease states by serving as the medication expert for "high cost, high touch" specialty medications. ${ }^{1}$ The Medical University of South Carolina (MUSC) currently has pharmacist involvement in 10 specialty clinics. Each pharmacist is focused on extensive medication education, patient adherence, medication-related side effects, and medication access or financial assistance if needed; however, the overarching goal is to improve patient outcomes through collaborative patient care.

Establishing a collaborative drug therapy management (CDTM) agreement with physicians allows a pharmacist to work directly with patients to optimize their medication regimens, order and assess required laboratory test monitoring, and evaluate potential treatment-related adverse events. This heightened monitoring is especially crucial for high-risk specialty medications, which may be part of a risk evaluation and mitigation strategy (REMS) program or require frequent safety monitoring. ${ }^{2}$ Physicians also benefit from additional pharmacist assistance, since they are able to effectively and safely care for a higher number of patients in a team-based setting.

The MUSC neurology clinic CDTM agreement allows pharmacists to assist in the management of complex disease states, including multiple sclerosis, movement disorders, migraine, and epilepsy. To describe the impact of pharmacist involvement in an ambulatory neurology clinic at MUSC, this study sought to evaluate how the addition of pharmacist services under a CDTM agreement affects clinic adherence to manufacturer-recommended laboratory test monitoring. Monitoring adherence focused on medications prescribed for multiple sclerosis (ie, dalfampridine, dimethyl fumarate, fingolimod, and teriflunomide) and epilepsy (ie, cannabidiol).

\section{Methods}

This study was a retrospective chart review of patients at a single academic medical center before and after the incorporation of pharmacy services in a neurology clinic. At MUSC, research with the intent of quality improvement or institutional program evaluation is exempt from institutional review board approval.

Patients were selected for inclusion if their medication lists included dalfampridine, dimethyl fumarate, fingolimod, or teriflunomide between July 1, 2014, and April 30, 2020, or included cannabidiol between July 1, 2018, and October 31, 2019; the authorizing provider's specialty listed on each medication order was "neurology," ensuring that all patients included were seen and evaluated by a provider in the neurology clinic. Included patients were required to live within the Charleston Tri-County Area, which includes Charleston, Berkeley, and Dorchester counties of South Carolina. ZIP codes for patients included in the analysis are listed in Supplementary Table 1 (available in online article).

Patients were excluded if the authorizing provider name for each order was "historical provider," which indicated that the medication was added to the patient's medication list based on patient report but was never prescribed by an MUSC provider.

For dalfampridine, dimethyl fumarate, fingolimod, and teriflunomide orders, patients were divided into prepharmacist and post-pharmacist groups. Patients in the pre-pharmacist group had medications prescribed with order dates between July 1, 2014, and September 30, 2017; this time frame was selected because the neurology clinic pharmacist joined the practice in October 2017. Patients in the post-pharmacist group had medications prescribed with order dates between November 1, 2019, and April 30, 2020; this time frame was selected because the neurology clinic prescribers formally approved pharmacist activities under a CDTM agreement in October 2019.

Because Epidiolex (cannabidiol) was approved by the US Food and Drug Administration on June 25, 2018, and laboratory adherence data are not available before a pharmacist was present in the neurology clinic, all patients with cannabidiol on their medication lists who met inclusion criteria as previously outlined were included for the purposes of descriptive analysis.

Patient charts were reviewed to determine if required laboratory test monitoring had been conducted as specified by the manufacturer's package insert. Laboratory tests were considered baseline if obtained on the date that the medication was first ordered or within 6 months before the prescription date. For medications with recommended laboratory test monitoring that did not include a prespecified stop date for such monitoring, laboratory test adherence was evaluated for the duration of medication use.

For patients taking medications before establishing care with an MUSC provider, baseline laboratory tests were considered "not applicable" and not included in adherence calculations. For patients who had medications discontinued before the maximum allowable time point of routine laboratory test monitoring per recommendations or who were lost to follow-up, maintenance labs were considered "not applicable" and not included in adherence calculations.

Percentage of adherence was calculated for each medication and laboratory test by dividing the number of patients with recommended laboratory test monitoring by the total number of applicable patients for that laboratory test monitoring. Descriptive statistics were calculated for all variables. Data are presented as median (interquartile range 


\section{FIGURE 1 Patient Attrition for Dalfampridine, Dimethyl Fumarate, Fingolimod, and Teriflunomide}

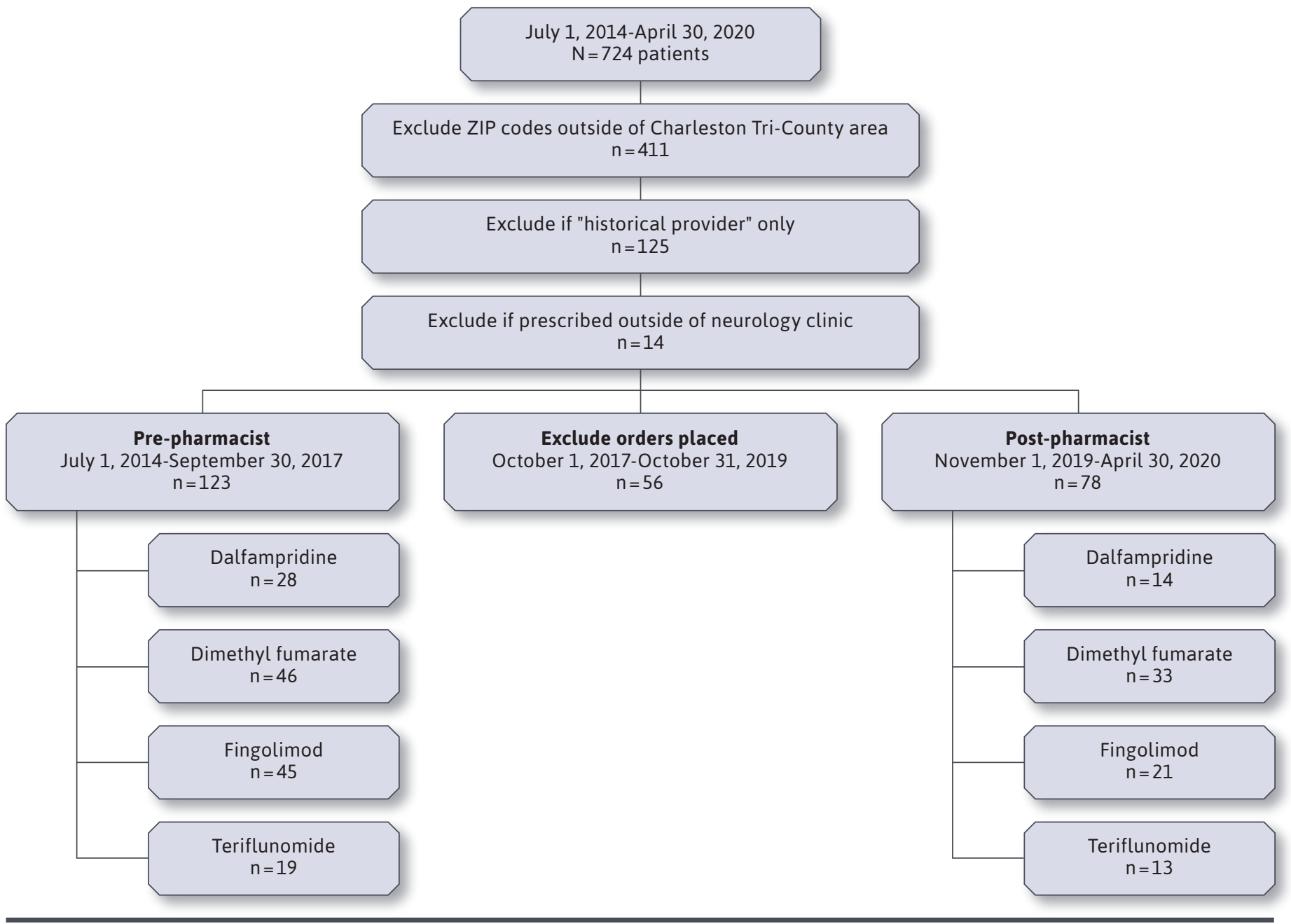

[IQR]) for continuous data and $n(\%)$ for frequency data. The difference between pre- and post-pharmacist group adherence was compared using chi-square or Fisher's exact tests. A value of $P<0.05$ was considered statistically significant.

\section{Results}

Between July 1, 2014, and April 30, 2020, there were 4,216 medication orders documented for dalfampridine, dimethyl fumarate, fingolimod, and teriflunomide within the electronic medical record (EMR) of MUSC for 724 unique patients. After applying inclusion and exclusion criteria, 123 unique patients with 138 medication orders were included in the pre-pharmacist group, and 78 unique patients with
81 medication orders were included in the post-pharmacist group. Patient inclusion in the pre- and post-pharmacist groups was not mutually exclusive, since some patients might have been on multiple medications.

Figure 1 shows a breakdown of patient attrition. Baseline characteristics by medication for patients in the prepharmacist and post-pharmacist groups are shown in Table 1. The median (IQR) age of included patients was 47 (38-57) years. The majority of patients were female, Caucasian, and taking the indicated medication for more than 6 months.

Between July 1, 2018, and October 31, 2019, there were 1,000 medication orders documented for cannabidiol within the EMR of MUSC for 497 unique patients. After applying 


\section{TABLE 1}

\section{Baseline Character
or Teriflunomide}

\begin{tabular}{|c|c|c|c|c|c|c|c|c|c|c|c|c|c|c|}
\hline & \multirow[b]{2}{*}{$\begin{array}{c}\text { Total } \\
\mathrm{N}=219\end{array}$} & \multicolumn{3}{|c|}{ Dalfampridine } & \multicolumn{4}{|c|}{ Dimethyl fumarate } & \multicolumn{4}{|c|}{ Fingolimod } & \multicolumn{2}{|c|}{ Teriflunomide } \\
\hline & & \multicolumn{2}{|c|}{$\begin{array}{c}\text { Before } \\
n=28\end{array}$} & $\begin{array}{l}\text { After } \\
\mathrm{n}=14\end{array}$ & \multicolumn{2}{|c|}{$\begin{array}{l}\text { Before } \\
n=46\end{array}$} & \multicolumn{2}{|c|}{$\begin{array}{l}\text { After } \\
\mathbf{n}=33\end{array}$} & \multicolumn{2}{|c|}{$\begin{array}{c}\text { Before } \\
n=45\end{array}$} & \multicolumn{2}{|c|}{$\begin{array}{l}\text { After } \\
\mathrm{n}=\mathbf{2 1}\end{array}$} & $\begin{array}{c}\text { Before } \\
n=19\end{array}$ & $\begin{array}{l}\text { After } \\
\mathrm{n}=13\end{array}$ \\
\hline Age, median (IQR) & $47(38-57)$ & 48.5 & $6-59)$ & $57(44-71)$ & 46.5 & $5-56)$ & 44( & 8-51) & 41 & $6-55)$ & 45( & 9-49) & $51(48-57)$ & $60(53-62)$ \\
\hline Female, \% (n) & $75.8(166)$ & 71.4 & (20) & $78.6 \quad(11)$ & 71.7 & (33) & 75.8 & (25) & 75.6 & (34) & 85.7 & (18) & $78.9 \quad(15)$ & $76.9 \quad(10)$ \\
\hline Caucasian, \% (n) & $64.8(142)$ & 80.0 & $(16)$ & $78.6 \quad(11)$ & 52.2 & (24) & 57.7 & (19) & 71.1 & (32) & 76.2 & (16) & 73.7 & 76.9 \\
\hline $\begin{array}{l}\text { African American, } \\
\% \text {, (n) }\end{array}$ & $31.5 \quad(69)$ & 35.7 & (10) & 14.3 & 45.7 & (21) & 39.4 & (13) & 26.7 & (12) & 23.8 & (5) & 21.1 & 15.4 \\
\hline $\begin{array}{l}\text { On treatment } \\
>6 \text { months, \% (n) }\end{array}$ & $85.8(188)$ & 82.1 & (23) & $85.7 \quad(12)$ & 82.6 & (38) & 75.8 & (25) & 95.6 & (43) & 90.5 & (19) & $84.2 \quad(16)$ & $92.3 \quad(12)$ \\
\hline
\end{tabular}

$I Q R=$ interquartile range.

inclusion and exclusion criteria, 51 unique patients were included for analysis. Figure 2 shows a breakdown of patient attrition. Baseline characteristics are shown in Table 2. The median (IQR) age of included patients was 19 (13-27) years. The majority of patients were male, Caucasian, and taking cannabidiol for more than 6 months.

Adherence to required laboratory test monitoring for all medications is shown in Table 3. Adherence improved in the post-pharmacist group for all monitoring points evaluated. For patients taking fingolimod, adherence in the post-pharmacist group significantly improved for "hepatic function tests every 6-9 months" $(P=0.005)$, "CBC every 6-9 months" ( $P=0.01)$, and "VZV IgG titer at baseline" $(P=0.005)$. A detailed breakdown of clinic adherence for each medication is shown in Supplementary Table 2 (available in online article).

\section{Discussion}

Pharmacists are increasingly being recognized for their contributions to patient care in neurology clinics. As early as 2002, Ryan et al described the successful piloting of an adverse drug reaction (ADR) surveillance program in a university-affiliated neurology clinic. ${ }^{8}$ The pharmacist was crucial to implementation of the program by providing staff education and further patient analysis when an ADR was identified. In 2012, Swain described taking on a consultant role in a neurology clinic that focused on patients with dementia or movement disorders. ${ }^{9}$ Patients were referred by a provider or self to have an appointment with the pharmacist for medication reconciliation and counseling. The most common recommendations made by the pharmacist were to discontinue, add, substitute, or change the dose of a medication, although such recommendations were often related to non-neurology medications. Similar interventions were made in a pharmacist-led neurology clinic for Veterans Affairs patients with headache, neuropathy, seizures, Parkinson disease, and non-Parkinson tremor. ${ }^{10}$ More recently, Eshiet et al demonstrated the positive impact of pharmacists on the management of patients with epilepsy by describing a clinical pharmacist-provided educational treatment program that led to improved patient knowledge and perception of epilepsy. ${ }^{11}$

Initial efforts of pharmacy services have resulted in highly integrated, multidisciplinary care systems that place pharmacists directly in clinics working along with other health care providers to assist with medication selection, education, monitoring, and resolution of financial concerns. ${ }^{2,12,13}$ Integrated models using specialty pharmacy services have demonstrated benefits, including decreased time to medication initiation and increased medication access, as well as improved financial assistance and patient adherence. $^{12}$

However, our study was the first to quantify the pharmacist impact on adherence to laboratory test monitoring. In our study, adherence rates improved for all required laboratory tests after a clinical pharmacist joined the neurology clinic practice; we believe this is because of the increased vigilance in ensuring that necessary laboratory tests were ordered and that results were evaluated before processing new prescriptions or refilling maintenance prescriptions.

Adherence rates were highest for common baseline laboratory tests, such as complete blood count (CBC), basic metabolic panel (BMP), and liver function tests. This higher rate may be due to overlap in required monitoring for other medication therapies or concomitant disease states. 


\section{FIGURE 2 Patient Attrition for Cannabidiol}

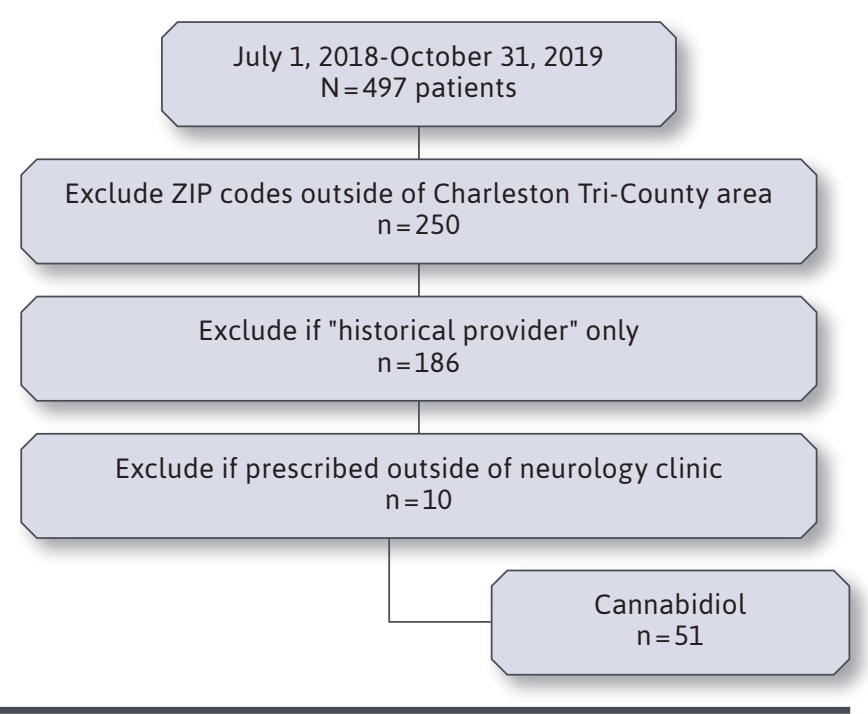

Adherence rates were lower for laboratory tests requiring annual or semiannual monitoring-obtaining repeat laboratory tests may be difficult if patients are unwilling to return to the clinic, which could be the result of perceived control of the disease state or lack of understanding of the benefit of monitoring. Adherence rates were lowest for those tests that are less commonly used in routine practice, such as the VZV IgG titer and tuberculosis screening.

Obtaining and reviewing required laboratory test monitoring with patients and providers is a significant opportunity for pharmacist involvement, given the high risk of potential adverse events and complex monitoring requirements for medications included in this analysis. ${ }^{14}$ For example, fingolimod requires a 6-hour first dose observation because of its potential for bradycardia and heart failure ${ }^{6}$; the coordination of this appointment along with ensuring that baseline ophthalmic exams and VZV antibody testing are completed requires great oversight and may become burdensome for other members of the multidisciplinary team.

Furthermore, specialty medications may require prior authorization before a patient's insurance company will help cover the cost of medications. Pharmacists can quickly demonstrate financial benefits to the health care system by getting involved in the prior authorization process and ensuring that patients have the necessary lab tests completed to meet payer expectations..$^{15}$ As newer therapies continue to become available for neurologic conditions,
TABLE 2 Baseline Characteristics for Patients Taking Cannabidiol

\begin{tabular}{l|cc}
\hline & \multicolumn{2}{|c}{ Cannabidiol, $\mathbf{n = 5 1}$} \\
\hline Age, median (IQR) & $19 \quad(13-27)$ \\
\hline Female, \% (n) & $43.1 \quad(22)$ \\
\hline Caucasian, \% (n) & 74.5 & $(38)$ \\
\hline African American, \% (n) & $19.6 \quad(10)$ \\
\hline On treatment >6 months, \% (n) & 68.6 \\
\hline IQR=interquartile range.
\end{tabular}

pharmacy involvement will be crucial and an invaluable asset to the health care provider.

Beyond the clinical benefits of having pharmacy services in the neurology clinic setting, the presence of a pharmacist can improve the satisfaction of patients and fellow health care team members..$^{9,16}$ Pharmacists may have the flexibility to spend greater amounts of time with patients during an appointment, allowing for increased patient satisfaction and understanding of their health care. ${ }^{9}$ Pharmacists can work closely with nursing to help share the responsibility of reviewing medication histories and monitoring adherence and adverse events. ${ }^{17}$ Pharmacy presence may also allow physicians more time to see new or more highly complex patients. ${ }^{12}$ Having a colleague to discuss particularly challenging cases and treatment decisions with can also decrease burnout and improve work-life balance for physicians. ${ }^{16}$ Finally, as health care continues to move toward quality-based care, pharmacists are held more accountable for medication- and patient-related outcomes of care. ${ }^{18}$ This increased focus on quality improvement by pharmacy may further relieve other health care providers from meeting such metrics alone. For example, pharmacists are considered eligible providers to address 5 of the 6 multiple sclerosis measures published by the American Academy of Neurology. ${ }^{19}$ Notably, pharmacist focus on laboratory monitoring adherence may assist in meeting the "Disease Modifying Therapies (DMT) Monitoring for Patients with Multiple Sclerosis (MS)" metric.

\section{LIMITATIONS}

Several limitations to our study exist. First, we did not seek to quantify the amount of time spent providing patient care, number of laboratory tests ordered, or number of recommendations made to the prescribers by the clinical pharmacist; thus, we cannot make conclusions regarding the impact made by the pharmacist directly. In addition, there is likely variation in the amount of time dedicated to 


\section{TABLE 3 Clinic Adherence to Required Lab Monitoring by Medication Before and After Pharmacist Incorporation Into Clinic ${ }^{3-7}$}

\begin{tabular}{|c|c|c|c|}
\hline Adherence, \% (n) & Before & After & $P$ value \\
\hline \multicolumn{4}{|l|}{ Dalfampridine } \\
\hline Metabolic panel at baseline & $68.2(15)$ & 83.3 & 0.64 \\
\hline Metabolic panel annually & $45.5(10)$ & $81.8 \quad(9)$ & 0.07 \\
\hline \multicolumn{4}{|l|}{ Dimethyl fumarate } \\
\hline Hepatic function tests at baseline (transaminases, bilirubin, alkaline phosphatase) & $77.8 \quad(28)$ & $89.5(17)$ & 0.47 \\
\hline CBC w/differential at baseline & $66.7(24)$ & $89.5(17)$ & 0.10 \\
\hline CBC w/differential every 6-12 months & $61.0(25)$ & 82.6 (19) & 0.10 \\
\hline \multicolumn{4}{|l|}{ Fingolimod } \\
\hline Hepatic function tests at baseline (transaminases, bilirubin) & $81.3 \quad(26)$ & $100.0 \quad(7)$ & 0.57 \\
\hline Hepatic function tests every 6-9 months & $43.9(18)$ & $84.2(16)$ & 0.005 \\
\hline $\mathrm{CBC}$ at baseline & $78.1(25)$ & $100.0 \quad(7)$ & 0.31 \\
\hline CBC every 6-9 months & $46.3 \quad(19)$ & $84.2(16)$ & 0.01 \\
\hline VZV IgG titer at baseline & $25.0 \quad(8)$ & $85.7 \quad(6)$ & 0.005 \\
\hline EKG at baseline & $87.5(28)$ & $100.0 \quad(7)$ & 1.00 \\
\hline Ophthalmology exam at baseline & 59.4 (19) & $85.7 \quad(6)$ & 0.39 \\
\hline Ophthalmology exam annually & $16.7 \quad(6)$ & $36.8 \quad(7)$ & 0.09 \\
\hline \multicolumn{4}{|l|}{ Teriflunomide } \\
\hline Hepatic function tests at baseline (transaminases, bilirubin) & $93.3(14)$ & 100.0 & 1.00 \\
\hline Hepatic function tests every month for 6 months & $13.3 \quad(2)$ & $66.7 \quad(2)$ & 0.11 \\
\hline CBC at baseline & $86.7(13)$ & $100.0 \quad(3)$ & 1.00 \\
\hline Tuberculosis screening (either blood or skin) at baseline & $6.7 \quad(1)$ & $66.7 \quad(2)$ & 0.06 \\
\hline \multicolumn{4}{|l|}{ Cannabidiol } \\
\hline Hepatic function tests at baseline (transaminases, bilirubin) & & 76.5 (39) & \\
\hline Hepatic function tests at 1-3 months & & $55.8(24)$ & \\
\hline Hepatic function tests at 4-6 months & & $62.2(23)$ & \\
\hline Hepatic function tests at 7-12 months & & $74.2(23)$ & \\
\hline
\end{tabular}

each patient by the pharmacist due to having only 1 pharmacist in a clinic with multiple providers.

Second, we did not evaluate clinical outcomes and cannot comment whether increased laboratory test monitoring adherence improves such outcomes. Third, we evaluated laboratory test monitoring adherence for the duration of therapy when applicable, but the reality of real-world clinical practice is that such monitoring is likely to relax as patients' duration of tolerated therapy increases. Fourth, our study has an overall small sample size; expanding included patients beyond the Charleston Tri-County area may further demonstrate improvements in clinic adherence.
Finally, the final 2 months of this study took place during the onset of the COVID-19 pandemic, when many patients chose to delay clinic appointments and our institution's pharmacy department implemented work-from-home policies; the impact that this situation may have had on our study is unknown.

\section{Conclusions}

Incorporation of a clinical pharmacist within a multidisciplinary neurology clinic improved adherence to manufacturer-recommended laboratory test monitoring. 


\section{DISCLOSURES}

No funding supported this study. The authors have nothing to disclose.

\section{REFERENCES}

1. American Pharmacists Association. Specialty pharmacy. Accessed March 2, 2020. https://www.pharmacist.com/ Practice/Patient-Care-Services/Specialty

2. Habibi M, Kuttab HM. Management of multiple sclerosis and the integration of related specialty pharmacy programs within health systems. Am J Health Syst Pharm. 2016;73(11):811-19.

3. Ampyra. Prescribing information. Acorda Therapeutics, Inc.; 2019. Accessed October 18, 2021. https://www. accessdata.fda.gov/drugsatfda docs/ label/2019/22250s017lbl.pdf

4. Aubagio. Prescribing information. Sanofi; 2019. Accessed October 18, 2021. https://www.accessdata.fda.gov/drugsatfda_docs/label/2019/202992s006lbl. pdf

5. Epidiolex. Prescribing information. Greenwich Biosciences, Inc.; 2018. Accessed October 18, 2021. https://www. accessdata.fda.gov/drugsatfda_docs/ label/2018/210365lbl.pdf

6. Gilenya. Prescribing information. Novartis; 2019. Accessed October 18, 2021. https://www.accessdata.fda.gov/drugsatfda docs/label/2019/022527s26lbl.pdf
7. Tecfidera. Prescribing information. Biogen; 2019. Accessed October 18, 2021. https://www.accessdata.fda.gov/drugsatfda_docs/label/2019/204063s024lbl. pdf

8. Ryan M, Gora-Harper ML, Caldwell F. Experience with an adverse drug reaction reporting program in a neurology specialty clinic. Ann Pharmacother. 2002;36(2):231-35.

9. Swain LD. A pharmacist's contribution to an ambulatory neurology clinic. Consult Pharm. 2012;27(1):49-57.

10. Martin AW, Heberle AP, Knight JM. Interventions associated with implementation of a pharmacist-led neurology pharmacotherapy clinic in an ambulatory care setting. J Am Coll Clin Pharm. 2019;2(2):116-22.

11. Eshiet U, Okonta J, Ukwe C. The efficacy of a pharmacist implemented educational treatment programme for people with epilepsy: a report of a randomised controlled trial. Seizure. 2019;69:147-53.

12. Bagwell A, Kelley T, Carver A, Lee JB, Newman B. Advancing patient care through specialty pharmacy services in an academic health system. J Manag Care Spec Pharm. 2017;23(8):815-20. doi: 10.18553/jmcp.2017.23.8.815

13. Hanson RL, Habibi M, Khamo N, Abdou S, Stubbings J. Integrated clinical and specialty pharmacy practice model for management of patients with multiple sclerosis. Am J Health Syst Pharm. 2014;71(6):463-69.
14. Rommer PS, Zettl UK, Kieseier B, et al. Requirement for safety monitoring for approved multiple sclerosis therapies: an overview. Clin Exp Immunol. 2014;175(3):397-407.

15. Leinss R, Karpinski T, Patel B. Implementation of a comprehensive medication prior-authorization service. Am J Health Syst Pharm. 2015;72(2):159-63.

16. Funk KA, Pestka DL, Roth McClurg MT, Carroll JK, Sorensen TD. Primary care providers believe that comprehensive medication management improves their work-life. J Am Board Fam Med. 2019;32(4):462-73.

17. Schultz J, Weber C, Kamholz J. Letter to the editor: The emerging role of pharmacists in the multidisciplinary care of patients with multiple sclerosis. Int J MS Care. 2016;18(5):219-20.

18. Andrawis M, Ellison C, Riddle S, et al. Recommended quality measures for health-system pharmacy: 2019 update from the Pharmacy Accountability Measures Work Group. Am J Health Syst Pharm. 2019;76(12):874-87.

19. American Academy of Neurology. Multiple Sclerosis Quality Measurement Set 2020 Update. November 2020. Accessed July 27, 2021. https://www.aan. com/siteassets/home-page/policy-andguidelines/quality/quality-measures/ multiple-sclerosis/2020-ms-quality-measurement-set.pdf 